\title{
The Cybathlon promotes the development of assistive technology for people with physical disabilities
}

\author{
Robert Riener ${ }^{1,2}$
}

\begin{abstract}
Background: The Cybathlon is a new kind of championship, where people with physical disabilities compete against each other at tasks of daily life, with the aid of advanced assistive devices including robotic technologies. The first championship will take place at the Swiss Arena Kloten, Zurich, on 8 October 2016.

The idea: Six disciplines are part of the competition comprising races with powered leg prostheses, powered arm prostheses, functional electrical stimulation driven bikes, powered wheelchairs, powered exoskeletons and brain-computer interfaces. This commentary describes the six disciplines and explains the current technological deficiencies that have to be addressed by the competing teams. These deficiencies at present often lead to disappointment or even rejection of some of the related technologies in daily applications.

Conclusion: The Cybathlon aims to promote the development of useful technologies that facilitate the lives of people with disabilities. In the long run, the developed devices should become affordable and functional for all relevant activities in daily life.
\end{abstract}

Keywords: Competition, Championship, Prostheses, Exoskeletons, Functional electrical stimulation, Wheelchairs, Brain computer interfaces

\section{Background}

Millions of people worldwide rely on orthotic, prosthetic, wheelchairs and other assistive devices to improve their qualities of life. In the US there live more than 1.6 million people with limb amputations [1] and the World Health Organization estimates the number of wheelchair users to about 65 million people worldwide [2]. Unfortunately, current assistive technology does not address their needs in an ideal fashion. For instance, wheelchairs cannot climb stairs, arm prostheses do not enable versatile hand functions, and power supplies of many orthotic and prosthetic devices are limited. There is a need to further push the development of assistive devices by pooling the efforts of engineers and clinicians to develop improved technologies, together with the feedback and experiences of the users of the technologies.

\section{Correspondence: riener@hest.ethz.ch}

'Sensory-Motor Systems Lab, ETH Zurich, Tannenstrasse 1, 8092 Zurich, Switzerland

${ }^{2}$ Spinal Cord Injury Center, University Hospital Bagrist, University of Zurich, Zurich, Switzerland
The Cybathlon is a new kind of championship with the aim of promoting the development of useful technologies. In contrast with the Paralympics, where parathletes aim to achieve maximum performance, at the Cybathlon, people with physical disabilities compete against each other at tasks of daily life, with the aid of advanced assistive devices including robotic technologies. Most current assistive devices lack satisfactory function; people with disabilities are often disappointed, and thus do not use and accept the technology. Rejection can be due to a lack of communication between developers, people with disabilities, therapists and clinicians, which leads to a disregard of user needs and requirements. Other reasons could be that the health status, level of lesion or financial situation of the potential user are so severe that she or he is unable to use the available technologies. Furthermore, barriers in public environments make the use of assistive technologies often very cumbersome or even impossible.

Six disciplines are part of the competition, addressing people with either limb paralysis or limb amputations. 
The six disciplines comprise races with powered leg prostheses, powered arm prostheses, functional electrical stimulation (FES) driven bikes, powered wheelchairs and powered exoskeletons (Fig. 1). The sixth discipline is a racing game with virtual avatars that are controlled by brain-computer interfaces (BCI). The functional and assistive devices used can be prototypes developed by research labs or companies, or commercially available products. The competitors are called pilots, as they have to control a device that enhances their mobility. The teams each consist of a pilot together with scientists and technology providers, making the Cybathlon also a competition between companies and research laboratories. As a result there are two awards for each winning team in each discipline: a medal for the person who is controlling the device and a cup for the provider of the device (i.e. the company or the lab).

A rehearsal was organized in July 2015 to test race tracks, scoring system and logistics and to generate footage for advertisement. The premiere will take place at the Swiss Arena, in Kloten, Zurich, on 8 October 2016. More information can be found on the Cybathlon website www.cybathlon.com.

\section{The six disciplines and the challenges in their fields}

Pilots with above knee amputations will use actuated prosthetic devices and compete along an obstacle course containing ramps, stairs, doors, soft-cushioned seats, barriers etc. Most of the commercially available leg prosthesis technologies are passive ballistic devices, which are easy to control but make uphill walking and stair ascent challenging. In persons with intact legs, especially the knee joint requires much higher capability of joint power generation during ascent than during level walking [3]. Consequently, users of passive prostheses, including microprocessorcontrolled dissipative knee prostheses, have to use hand rails and/or perform an asymmetric non-physiological gait to compensate for the missing power generation in the knee (see for example [4] among many other studies). Powered leg prostheses can induce the missing power and in this way solve these deficiencies; however, the control of such devices is not trivial when interfacing them with the user's motion intention [5]. Additionally, state-of-theart batteries are either too heavy or lack sufficient capacity to provide power throughout an entire day. The teams at the Cybathlon will showcase new technologies that might overcome current deficiencies.

At the Cybathlon, pilots with amputations of the lower arm or above will use actuated prosthetic hands and arms to complete various household and food preparation tasks (Fig. 2). The dexterity and versatility of currently available prosthetic hand devices is usually limited with respect to the number of grasps and tasks that can be successfully performed. Therefore, persons with unilateral amputations use their intact arm to perform most daily tasks. Bimanual tasks, which require a high load transfer (e.g., carrying a heavy box) or particular fine motor skills (e.g., opening a small jar of jam) are challenging, because they cannot be solved with most state-of-the-art upper arm prostheses. Consequently, up to $60 \%$ of people with upper-limb amputation fitted for conventional upper-limb prosthetic device fail to use it regularly or reject it altogether $[6,7]$. The high rejection rate of upper limb prostheses has been attributed to poor training, late fitting, limited usefulness especially for the users with more proximal amputations, and various other factors. Significantly lower rates of rejection can be seen for more advanced, i.e. body-powered $(26 \%)$ and electric $(23 \%)$ devices [8].

Four out of the six disciplines of the Cybathlon address people with limb paralysis of varying degrees after lesions such as spinal cord injury: Pilots with complete paraplegia will compete in a bike race, where FES devices assist them in performing pedaling movements. FES technology has been used for movement restoration for decades, but has not achieved satisfactory performance due to limitations in setup-time, movement controllability, muscle force magnitude, muscle selectivity and fatigue resistance $[9,10]$. Most promising stimulation systems are implanted, as they yield better selectivity and higher force output than non-invasive systems $[9,11]$. However, there are drawbacks with respect to invasiveness, risk of infections and costs. Because of these deficiencies, current FES technology has not been

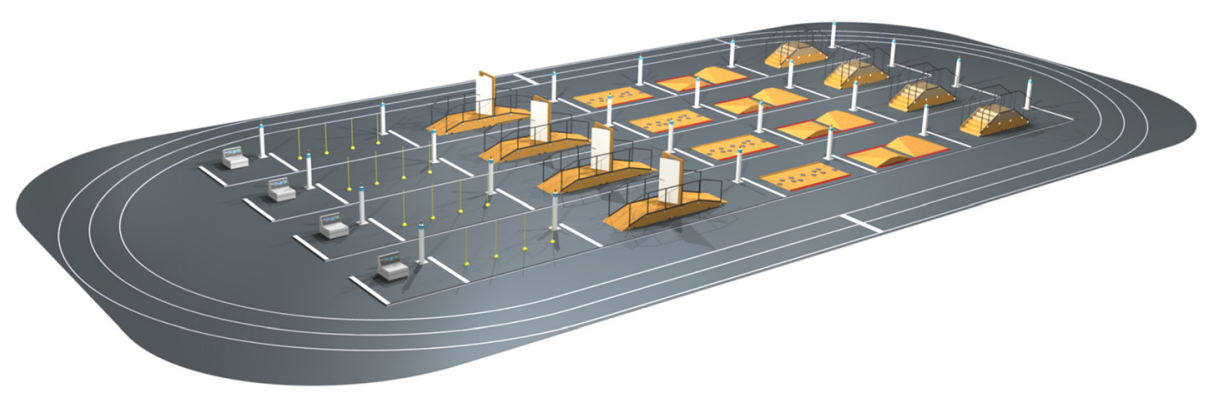

Fig. 1 Arena with four parallel race tracks designed for the exoskeleton competition. The pilots start at the left and have to overcome six obstacles with increasing difficulty level 


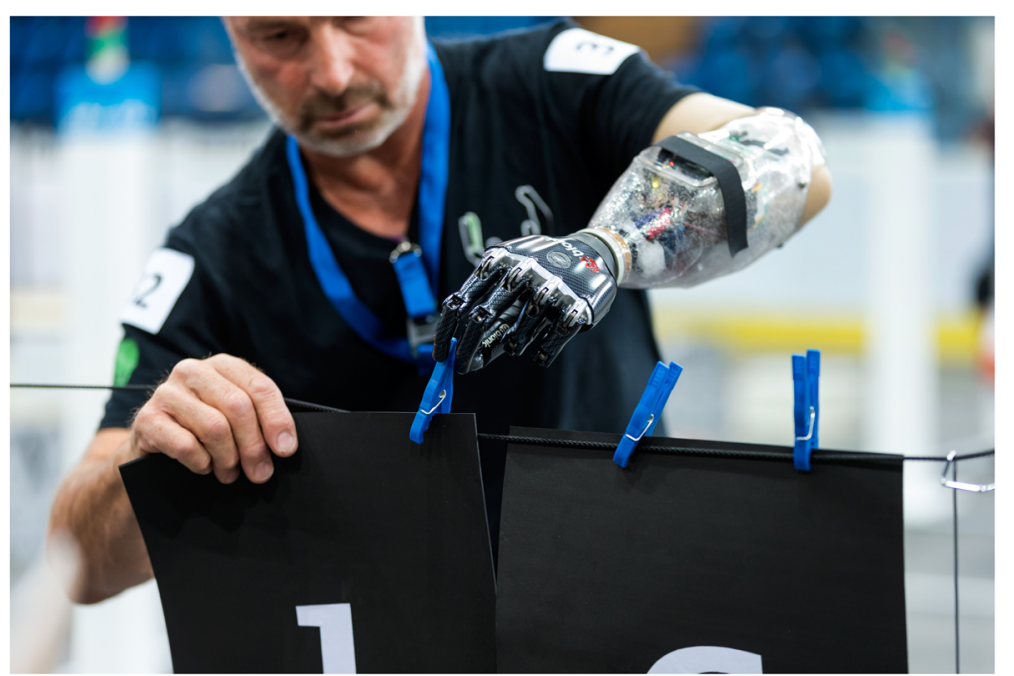

Fig. 2 Pilot with a powered arm prosthesis performing a daily living task. Picture was taken at the Cybathlon rehearsal in July 2015 by Alessandro Della Bella, ETH Zurich. The pilot on this image as consented to the publication of this image

accepted by physicians and patients for daily clinical routine [12].

In both the powered wheelchair race and the powered exoskeleton race, pilots with paralysis will master obstacle courses with ramps, stairs, bends, doors and uneven terrain (Fig. 1). More and more companies offer advanced and powerful solutions for wheelchairs. However, control technology does not provide adequate mobility and comfort for many electrically powered wheelchair users, especially under adverse driving conditions [13]. Wheelchair accessibility in public buildings is still limited despite the enforcement of existing laws and regulations [14]. Most outdoor devices are too bulky and not agile enough for indoor use, whereas commercial indoor wheelchairs are not capable of overcoming uneven terrain or steps. So called intelligent or smart wheelchairs have been available for decades, but have not yet been adopted by a large portion of the population $[15,16]$. An alternative to wheelchairs are exoskeletal devices that assist people with paraplegic lesions during gait in the upright position $[17,18]$. However, battery power is limited to a few hours of operation and the devices are still very bulky and heavy. Most of the commercially available multi-joint exoskeletons have weights in the range of 21-28 kg, with the device "REX" reaching a weight of almost $40 \mathrm{~kg}[17,19]$. Furthermore, current commercial systems have a limited number of degrees of freedom and reduced ranges of movements preventing the devices from gait on inclined surfaces or stairs. Thus, exoskeletal devices are not yet a realistic alternative for lightweight, energy efficient, and often foldable manual wheelchairs.

In the BCI race, pilots with paralysis of all four limbs will control a virtual avatar in a racing game displayed on a computer screen. The best pilots will be able to distinguish three different commands to overcome three different kinds of virtual obstacles and, thus, will be rewarded by a temporal advantage in the game. A wrong command or a command with too long latency will be penalized by decelerating the avatar on its track. BCI technology is becoming more and more popular, however most systems only function accurately in a lab environment [20]. The time needed for device setup, comfort, cosmetic aspects, function and reliability are still not satisfactory and have prevented broad use and acceptance outside labs [21].

\section{Conclusion}

The Cybathlon will provide a platform that encourages exchange between people with disabilities or physical weaknesses, the research and development world, funding agencies, and the general public. In this way, the Cybathlon aims to promote the development of useful technologies that facilitate the daily lives of people with disabilities or physical weaknesses and provide the basis for more independence. In the long run, the developed devices should become affordable and functional for all relevant activities in daily life.

Cybathlon can also be considered as a complement to the Olympic or Paralympic games. In contrast to the Paralympic games, it allows the use of any kind of technical aids, thus also enabling people with more severe disabilities to participate in a competition. The goal is not to be the fastest and the strongest among the participants, rather the goal is to be the most skilled pilot who utilizes advanced technologies in ways that allow the challenges of everyday life to be overcome with ease. 


\section{Abbreviations}

BCl, Brain-Computer Interface; FES, Functional Electrical Stimulation; US, United States

\section{Acknowledgements}

Special thanks go to Roland Auberger Verena Klamroth-Maganska, Domen Novak, Anna Pagel, Serge Pfeifer, Kai Schmidt, Linda Seward, Roland Sigrist, Peter Wolf, Dario Wyss for their contributions to this manuscript, including organization of references and proof-reading.

\section{Funding}

This work is funded by ETH Zurich, Switzerland, and the Swiss National Science Foundation (SNSF) National Competence Center in Research (NCCR) in Robotics.

\section{Availability of data and materials}

Not applicable.

\section{Author's contribution}

$\mathrm{RR}$ is the inventor, initiator and chief organizer of the Cybathlon. He has drafted the manuscript.

\section{Competing interests}

Cybathlon is a non-profit, charitable event. The author is initiator of the Cybathlon and member of the organizing board. The author confirms that there are no further competing interests.

\section{Ethics approval and consent to participate}

The individual in Fig. 2 consented to the publication of this image.

\section{Received: 4 April 2016 Accepted: 23 May 2016}

\section{Published online: 31 May 2016}

\section{References}

1. Ziegler-Graham K, MacKenzie EJ, Ephraim PL, Travison TG, Brookmeyer R. Estimating the Prevalence of Limb Loss in the US. Arch Phys Med Rehabil. 2008:89:422-9.

2. World Health Organization. Guidelines on the provision of manual wheelchairs in less resourced settings. WHO Press, 2008. http://www.who. int/disabilities/publications/technology/wheelchairguidelines/en/.

3. Riener R, Rabuffetti M, Frigo C. Stair ascent and descent at different inclinations. Gait Posture. 2002;15:32-44.

4. Schaarschmidt M, Lipfert SW, Meier-Gratz C, Scholle H-C, Seyfarth. Functional gait asymmetry of unilateral transfemoral amputees. Hum Mov Sci. 2012;31:907-17.

5. Novak D, Riener R. A survey of sensor fusion methods in wearable robotics. Robot Auton Syst. 2014;73:155-70.

6. Wright TW, Hagen AD, Wood MB. Prosthetic usage in major upper extremity amputations. J Hand Surg [Am]. 1995;20:619-22.

7. Dudkiewicz I, Gabrielov R, Seiv-Ner I, Zelig G, Heim M. Evaluation of prosthetic usage in upper limb amputees. Disabil Rehabil. 2004;26:60-3.

8. Biddiss EA, Chau T. Upper limb prosthesis use and abandonment: a survey of the last 25 years. Prosthetics Orthot Int. 2007;31:236-57.

9. Hardin E, Kobetic R, Murray L, Corado-Ahmed M, Pinault G, Sakai J, Bailey SN, Ho C, Triolo RJ. Walking after incomplete spinal cord injury using an implanted FES system: A case report. JRRD. 2007:44:333-46

10. J. del-Ama A, Koutsou AD, Moreno JC, de-los-Reyes A, Gil-Agudo A, Pons JL. Review of hybrid exoskeletons to restore gait following spinal cord injury. JRRD. 2012:49:497-514

11. Agarwal S, Triolo RJ, Kobetic R, Miller M, et al. Long-term user perceptions of an implanted neuroprosthesis for exercise, standing, and transfer after spinal cord injury. JRRD. 2003;40:241-52.

12. Nightingale EJ, Raymond J, Middleton JW, Crosbie J, Davis GM. Benefits of FES gait in a spinal cord injured population. Spinal Cord. 2007:45:646-57.

13. Ding D, Cooper RA. Electric Powered Wheelchairs. IEEE Control Systems Magazine. 2005;25(2):22-34.

14. Welage N, Liu KPY. Wheelchair accessibility of public buildings: a review of the literature. Disabil Rehabil Assist Technol. 2011;6:1-9.

15. Simpson RC. Smart wheelchairs: a literature review. JRRD. 2005;42:423-36.

16. Garcia JC, Marron M, Urena J, Gualda D. In: Encarnaçao P, Azevedo L, Gelderblom GJ, Newell A, Mathiassen N-E, editors. Assistive Technology:
From Research to Practice. Amsterdam, The Netherlands: IOS Press BV; 2013. p. 202-9.

17. Riener R. Rehabilitation robotics. Foundations and Trends in Robotics. 2012; 3:1-137.

18. Chen G, Chan CK, Guo Z, Yu H. A review on lower extremity assistive robotic exoskeleton in rehabilitation therapy. Crit Rev Biomed Eng. 2013. doi:10.1615/CritRevBiomedEng.2014010453.

19. Lajeunesse V, Vincent C, Routhier F, Careau E, Michaud F. Exoskeletons' design and usefulness evidence according to a systematic review of lower limb exoskeletons used for functional mobility by people with spinal cord injury. Disabil Rehabil Assist Technol. 2015:4:1-13.

20. Nicolas-Alonso LF, Gomez-Gil J. Brain computer interfaces, a review. Sensors. 2012:12:1211-79.

21. Shih JJ, Krusienski DJ, Wolpaw JR. Brain-computer interfaces in medicine. Mayo Clin Proc. 2012:87:268-79.

\section{Submit your next manuscript to BioMed Central and we will help you at every step:}

- We accept pre-submission inquiries

- Our selector tool helps you to find the most relevant journal

- We provide round the clock customer support

- Convenient online submission

- Thorough peer review

- Inclusion in PubMed and all major indexing services

- Maximum visibility for your research

Submit your manuscript at www.biomedcentral.com/submit
Biomed Central 